\section{POS0293 TRHEUMA REGISTRY PROVIDES FIRST EVIDENCE OF DIFFERENT COURSE OF RHEUMATIC IMMUNE- RELATED ADVERSE EVENTS AND TUMOUR RESPONSE RATES DEPENDING ON THE TUMOUR ENTITY}

L. Diekmann ${ }^{1}$, L. Daniello², J. Kunz ${ }^{2}$, J. Leipe ${ }^{3}$, H. M. Lorenz ${ }^{1}$, J. Hassel ${ }^{4}$, K. Jordan ${ }^{1}$, P. Christopoulos ${ }^{2}$, K. Benesova ${ }^{1} .{ }^{1}$ University Hospital Heidelberg, Department of Medicine V, Hematology, Oncology and Rheumatology, Heidelberg, Germany; ${ }^{2}$ Thoraxklinik Heidelberg, Department of Thoracic Oncology, Heidelberg, Germany; ${ }^{3}$ University Hospital Mannheim, Division of Rheumatology, Department of Medicine V, Mannheim, Germany; ${ }^{4}$ University Hospital Heidelberg, Department of Dermatology and National Center for Tumor Diseases, Heidelberg, Germany

Background: Rheumatic immune-related adverse events (irAE) are associated with a better tumour response to immune checkpoint inhibitors (ICI). In contrast to other irAEs, their potentially chronic course may require long-term immunosuppressive treatment.

Objectives: Our registry-based study analyses real-world data on the characteristics and outcome of rheumatic irAEs and underlying malignancy. Herein, we present first evidence that these parameters and the optimal clinical management may differ depending on the tumour entity.

Methods: The TRheuMa registry is a prospective long-term observational study of a patient cohort suffering from rheumatic side effects of cancer therapies with focus on ICl. It is part of the MalheuR project initiated in July 2018 at the University Hospital Heidelberg to explore interrelations of malignancies and RMDs. Results: 64 patients were recruited due to a rheumatic irAE under ICI treatment (nivolumab $n=30$, pembrolizumab $n=33$, ipilimumab $n=12$, PD-L1i $n=5$, ipi/nivo $n=10$ ) with a follow-up of up to 30 months. Of these, $47 \%$ had NSCLC and $41 \%$ melanoma. In local cohorts of patients receiving ICI, $4 \%$ of NSCLC ( $n$ total $=888$ ) and $13 \%$ of melanoma ( $n$ total $=195$ ) developed a rheumatic irAE. $7 \%$ of NSCLC and $23 \%$ of melanoma patients experienced a flare of a pre-existing RMD. De novo irAE mostly resembled phenotypes of spondyloarthritis both in NSCLC $(43 \%)$ as well as in melanoma patients $(33 \%)$. CRP levels were increased in $83 \%$ of NSCLC and $71 \%$ of melanoma patients. Almost all irAE patients showed autoantibody negativity and signs of inflammation in ultrasound examination $(96 \%)$. Comparison of best responses to treatment in patients with and without rheumatic irAE in melanoma and without any irAE in NSCLC patients were as following: Complete remission (CR) in $48 \%$ vs. $4 \%$ of melanoma patients and partial remission (PR) in $68 \%$ vs. $41 \%$ of NSCLC patients. In accordance with our severity-based treatment algorithm, $25 \%$ of the melanoma patients in CR and $16 \%$ of the NSCLC patients in PR needed add-on DMARDs for sufficient irAE-treatment. ICl-treatment was discontinued in 7 cases ( $17 \%$ NSCLC, $8 \%$ melanoma)

Conclusion: Prospective real-world data from the TRheuMa-registry provide first evidence that rheumatic irAE have distinct characteristics depending on the underlying malignancy. Oncological outcome was better with rheumatic irAE than in their absence and this effect was more pronounced in melanoma patients despite a larger use of immunosuppressants for irAE-treatment.

Disclosure of Interests: Leonore Diekmann: None declared, Lea Daniello: None declared, Julia Kunz: None declared, Jan Leipe Consultant of: Pfizer; Novartis; Honoraria (self), Abbvie; Astra Zeneca; BMS; Celgene; Hospira; Janssen-Cilag; Gilead; LEO Pharma; Lilly; MSD; Roche; Sanofi; UCB., Grant/research support from: Research grant/Funding (self): Pfizer; Novartis; Honoraria (self), Hanns-Martin Lorenz Consultant of: Abbvie; BMS; MSD; Pfizer; Celgene; Roche; Chugai; Medac; GSK; Honoraria (self), Novartis; UCB; Janssen-Cilag; Astra Zeneca; Lilly, Grant/research support from: Research grant/Funding (institution): Abbvie; BMS; MSD; Pfizer; Celgene; Roche; Chugai; Medac; GSK; Honoraria (self), Research grant/Funding (institution), Novartis; UCB; Janssen-Cilag; Astra Zeneca; Lilly; Research grant/Funding (institution): Baxter; SOBI; Biogen; Actelion; Mundipharma; Bayer Vital; Octapharm; Sanofi; Hexal; Thermo Fischer; Shire., Jessica Hassel Consultant of: MDS; Honoraria (self): Roche; Novartis; Pierre Fabre., Grant/research support from: BMS; Honoraria (self), Karin Jordan Consultant of: Advisory/Consultancy: Amgen; Merck; MSD; Riemser; Helsinn; Tesaro; Kreussler; Voluntis; Pfizer; Pomme-med; Hexal., Petros Christopoulos Consultant of: advisory board/lecture fees from AstraZeneca, Boehringer Ingelheim, Chugai, Novartis, Pfizer, Roche, Takeda., Grant/research support from: research funding from AstraZeneca, Novartis, Roche, Takeda, Karolina Benesova Grant/research support from: Foundations and Awards" commission of the University of Heidelberg: University of Heidelberg; AbbVie; Novartis; Rheumaliga Baden-Württemberg e.V DOI: 10.1136/annrheumdis-2021-eular.591

\section{POS0294 \\ PROFILE OF SPINAL TUMORS IN RHEUMATOLOGY DEPARTMENTS}

B. A. Hiba ${ }^{1}$, H. Sahli ${ }^{2}$, S. Boussaid ${ }^{3}$, S. Nouicer ${ }^{3}$, S. Jemmali ${ }^{3}$, S. Rekik², M. Eleuch ${ }^{1}{ }^{1}$ La Rabta, Rheumatology, Tunis, Tunisia; ${ }^{2}$ La Rabta, Rheumatology, Tunis, Tunisia; ${ }^{3}$ La Rabta, Rheumatology, Tunis, Tunisia
Background: Spine tumors remain a hot topic because of their associated morbidity by affecting motor and sensory function. Contrary to metastatic spine disease (MSD), extremely prevalent, rise within or surrounding the spinal cord and/or vertebral column, primary spinal tumors are rare, $5 \%$ of all primary skeletal tumors and frequently benign $(20 \%)$. The diagnostic delay of these tumors, even when benign, is associated with a poor prognosis. Establishing the correct diagnosis is heavily reliant on magnetic resonance imaging and histological confirmation.

Objectives: to provide an overview of the epidemiology, radiological and histopathological of spinal tumors diagnosed in a rheumatology department.

Methods: A retrospective study consisting of clinical characteristics analysis, laboratory and $\mathrm{x}$-ray examinations, was performed on 40 patients who were hospitalised for a spinal tumor, in a rheumatology department, over 5-year period from 2015 to 2020.

Results: A total of 40 patients with a mean age of $66 \pm 13.5$ years [18-93] and a sex-ratio of 1.1 , were included. The most common initial complaints were inflammatory back pain $(67.5 \%)$ and fatigue $(52.5 \%)$, with a median duration of 5 months. Physical examination abnormalities included lumbar stiffness $(32.5 \%)$, radicular signs $(18.7 \%)$, hepatomegaly $(12.5 \%)$, and lymphadenopathy $(17.5 \%)$. Neurological deficit was found in only 3 patients $(7.5 \%)$. Hypercalcemia (corrected serum calcium $>105 \mathrm{mg} / \mathrm{l}$ ), and anemia (hemoglobin $(\mathrm{Hb})<100 \mathrm{~g} / \mathrm{l}$ in female, $\mathrm{Hb}$ $<110 \mathrm{~g} / \mathrm{l}$ in male) were present initially in respectively $47.5 \%$ and $46.3 \%$ patients, while biological inflammatory syndrome was present in $89.7 \%$ patients (median C-reactive protein of 44.7). Tumor markers were performed in 12 patients and they were positive in 9 of them.

Plain radiographs findings were vertebral compression fractures $(43.6 \%)$, osteolytic lesions $(30.8 \%)$ and osteoblastic lesions $(12.5 \%)$. Lumbar spine was the most affected $(57.5 \%)$, followed by the dorsal spine $(45 \%)$. Magnetic resonance imaging (MRI) was performed in 55\%, and the most common lesion was low signal intensity on T1-weighted sequences and high signal intensity on T2-weighted sequences $(68.1 \%)$.

In our study, only one patient was diagnosed for a myxopapillary ependymoma, a benign primary spinal tumor characterised by a metastatic dissemination risk. For the rest (39 patients), the diagnosis of bone metastasis, multiple myeloma $(57.7 \%)$, and of solid tumor cancers $(40 \%)$, were established. Primary cancers were mainly prostate cancer $(37.5 \%)$, lung cancer $(18.7 \%)$ and kidney cancer (18.7\%). By a median follow-up time of 25 months, overall survival rate was $30 \%$. Conclusion: Extradural lesions are the most common, and are typically metastatic. Special attention should be pain to the patient's medical history and laboratory abnormalities. In fact, an early diagnosis requires a high index of clinical suspicion.

Disclosure of Interests: None declared

DOI: 10.1136/annrheumdis-2021-eular.3905

\section{\begin{tabular}{|l|l}
\hline POS0295 NO CAUSAL EFFECTS OF GENETICALLY \\
\hline
\end{tabular} DETERMINED SERUM URATE LEVELS ON THE RISK OF ALL-CAUSE AND SITE-SPECIFIC CANCER: A MENDELIAN RANDOMIZATION STUDY}

T. Fatima ${ }^{1}$, Y. Borné2 , M. Dehlin ${ }^{3}$, S. Burgess ${ }^{4}$, A. Mason ${ }^{5}$, L. T. H. Jacobsson ${ }^{6}$, M. C. Kapetanovic ${ }^{7}{ }^{1}$ University of Gothenburg, Department of Rheumatology and Inflammation Research, Göteborg, Sweden; ${ }^{2}$ University of Lund, Department of Clinical Sciences, Malmö, Sweden; ${ }^{3}$ University of Gothenburg, Department of Rheumatology and Inflammation Research, Gothenburg, Sweden; ${ }^{4}$ University of Cambridge, MRC Biostatistics Unit, School of Clinical Medicine, Cambridge, United Kingdom; ${ }^{5}$ University of Cambridge, Cardiovascular Epidemiology Unit, School of Clinical Medicine, Cambridge, United Kingdom; ${ }^{3}$ University of Gothenburg, Department of Rheumatology and Inflammation Research, Gothenburg, Sweden; ${ }^{7}$ University of Lund, Department of Clinical Sciences, Lund, Sweden

Background: Positive associations between urate levels and gout and the risk of some cancer types (urogenital, prostate, gastrointestinal and lung) have been reported in a number of observational studies; however, whether the relationship is causal remains uncertain.

Objectives: The study aim was to evaluate a causal effect of genetically determined serum urate (SU) concentrations on cancer risks (overall and major cancer types) in individuals with European ancestry using Mendelian randomization (MR) analyses design.

Methods: We used the individual-level data from two population-based Swedish cohorts including middle-aged subjects (mean follow-up $=21.2$ years), Malmö Diet Cancer and Malmö Preventive Project (MDC/MPP), for one-sample MR setting. Data from a total of 17,597 individuals $(n=17,597$ for SU at baseline, diagnoses during follow-up: 5659 for all-cause, 516 for bladder, 545 for lung, 791 for bowel, 1521 for prostate and 729 for breast cancer) was included. For two-sample MR, summary-statistic data for SU was obtained from Global Urate Genetic Consortium (GUGC: $n=110,347$ ), while UK-Biobank data was employed for several major cancer outcomes ( $n=36,815$ for all-cause, 2,245 for 